\title{
Chromosome 8q as the most frequent target for amplification in early gastric carcinoma
}

\author{
JI UN KANG

\begin{abstract}
Department of Biomedical Laboratory Science, Korea Nazarene University, Cheonan 330-718, Republic of Korea
\end{abstract}
Received July 12, 2013; Accepted January 15, 2014

DOI: $10.3892 / \mathrm{ol} .2014 .1849$

\begin{abstract}
Early gastric carcinoma (GC) is considered to be a curable cancer, as it progresses to the advanced stage following varying durations. Understanding the early stage of GC may provide an insight into its pathogenesis and contribute to reducing the mortality rate of this disease. To investigate the genomic aberrations associated with 22 cases of early GC, high-density microarray comparative genomic hybridization was performed in the present study. The most notable finding was copy number gains $\left(\log _{2}\right.$ ratio $\left.>0.25\right)$ on the long arm of chromosome 8 , which occurred in $77.3 \%$ (17/22) of GC cases, and the delineated minimal common region was 8q22.1-q24.3. More specifically, two amplified $\left(\log _{2}\right.$ ratio $\left.>1\right)$ loci in the 8q22.1-q24.3 region were detected in 18.2\% (4/22) of GC cases. The first loci covered a region of 102.4-107.9 kb, mapping on $8 \mathrm{q} 22.3-\mathrm{q} 23.1$, and comprised the transcription factor CP2-like 3 gene. The second loci, spanning 128.7-145.7 kb on $8 \mathrm{q} 24.21-\mathrm{q} 24.3$, comprised the representative oncogene of myelocytomatosis. Furthermore, the following possible target genes that were not previously considered to play a pathogenic role in GC were identified: Plasmacytoma variant translocation 1 , cysteine/histidine rich 1 , kinesin family member $\mathrm{C} 2$, forkhead box $\mathrm{H} 1$, protein phosphatase 1 regulatory subunit 16A, glutamic-pyruvate transaminase, LOC113655 and RecQ protein-like 4. In the present study, previous findings showing that $8 \mathrm{q}$ mutations accumulate early during the multistage pathogenesis of GC were confirmed and expanded upon. The confirmation of previously reported $8 \mathrm{q}$ gains and the identification of novel target genes at 8q22.1-q24.3 amplified chromosomal sites should aid in improving our understanding of the molecular mechanisms underlying the tumorigenesis of early GC.
\end{abstract}

Correspondence to: Professor Ji Un Kang, Department of Biomedical Laboratory Science, Korea Nazarene University, 456 Ssangyong, Seobuk, Cheonan, Chungnam 330-718, Republic of Korea

E-mail: jukang@kornu.ac.kr

Key words: gastric carcinoma, microarray CGH, copy number gains, high-level amplifications

\section{Introduction}

Eradication of early gastric carcinoma (GC) could contribute to a reduction in the mortality of GC, given that most early GCs progress to become advanced GCs (1). Therapeutic interventions for late-stage GC are usually limited to non-curative gastrectomy, lymphadenectomy and post-operative chemoradiotherapy (2). The five-year relative survival rates for GC patients are $<30 \%$ in the majority of countries (3). Hence, the identification of novel biomarkers is of great clinical importance for early diagnosis, targeted treatment and prognosis evaluation in GCs.

Gastric tumorigenesis is a heterogeneous process that occurs following a series of clonal molecular genetic alterations, including genomic gains and losses, particularly deletion of tumor-suppressor genes and amplification of oncogenes. Unveiling abnormalities of specific genes may offer novel insights into the mechanisms of local growth or the metastatic potential of early GCs, and allow patients to be stratified into different risk categories or be treated with novel options for targeted therapy (4).

Cytogenetic studies have been performed to evaluate genetic alterations associated with early GCs $(1,2,4,5)$. Defining the genetic instability aids in the identification of the tumor-specific signatures involved in the initiation and progression of GCs, and thus aids in locating genomic biomarkers for the early detection of GC (5). However, the molecular mechanism of GC development remains to be understood, and identification of the predictive markers in the early stage is crucial. There is a critical requirement for identifying biomarkers for early detection and novel treatments for GC. Therefore, in the present study, whole genome array comparative genomic hybridization (CGH) was conducted to investigate DNA copy number alterations and new candidate genes that may be indicative and specific for early GC.

\section{Materials and methods}

Study materials. A total of 22 gastric tumor samples were obtained from patients treated at the Department of General Surgery of Chungnam National University Hospital in Taejeon, South Korea. None of these patients had received pre-operative chemotherapy or radiation. The stage of disease was based on the tumor-node-metastasis classification using the Union International Cancer Center staging system. The original 
Table I. Various chromosomal recurrent minimal regions of genetic alterations on the long arm of chromosome 8 in 22 GCs.

\begin{tabular}{llll}
\hline Regions & \multicolumn{1}{c}{ Gene contained in clones } & \% of gains & \% of amplifications \\
\hline 8q21.1-q21.3 & IL7, PMP2, FABP9, FABP4, MMP16, NBS1, DECR1 & $22.7(5 / 22)$ & $18.2(4 / 22)$ \\
$8 q 22.1-q 23.3$ & TFCP2L3, MGC35555 & $77.3(17 / 22)$ & $9.1(2 / 22)$ \\
8q24.11-q24.3 & EXT1, NOV, LOC392264, MYC, PVT1, LOC401475, & & \\
& SIAT4A, WISP1, NDRG1, TG, LOC286094, NDRG1, & & \\
& LOC392271, COL22A1, CYHR1, KIFC2, FOXH1, & & \\
& PPP1R16A, GPT, LOC113655, RECQL4, ZC3HDC3, & & \\
& LOC389693, GSDMDC1, LOC389694, PP3856, EEF1D &
\end{tabular}

\begin{abstract}
${ }^{\mathrm{a}}$ Alterations were defined by $\log _{2}$ ratio thresholds of $>0.25$ for copy number gain. ${ }^{\mathrm{b}}$ Alterations were defined by $\log _{2}$ ratio thresholds of $1 \log _{2}$ ratio for high-level amplification. Genomic positions were retrieved from the UCSC Genome browser web page [http://genome.cse.ucsc.edu; Build 36, March 2006 version (hg18)]. GC, gastric carcinoma; UCSC, University of California Santa Cruz.
\end{abstract}

diagnostic material of all patients was reviewed to verify the original histopathological diagnosis and staging according to the World Health Organization classification system (6). The present study was reviewed and approved by the Institutional Review Board of the Chungnam National University Hospital, and written informed consent was obtained from each patient according to the institutional regulations.

Array-CGH experiment. DNA isolation was performed using a DNA isolation kit according to the manufacturer's instructions (Promega, Madison, WI, USA), with certain modifications as previously described $(7,8)$. Array-CGH was conducted using the MacArray ${ }^{\mathrm{TM}}$ Karyo 1.4 K BAC-chip (Macrogen, Seoul, Korea) (9-11) according to the manufacturer's instructions and as described in our previous studies $(12,13)$. Briefly, all clones were two-end sequenced using an ABI PRISM 3700 DNA Analyzer (Applied Biosystems, Foster City, CA, USA), and their sequences were blasted (BLAST; http://blast.ncbi.nlm.nih.gov/Blast.cgi).

Hybridizations were carried out using a standard direct method as previously described $(14,15)$. Briefly, $500 \mathrm{ng}$ normal male DNA (reference) and digested tumor DNA (test) was labeled with Cy5-dCTP and Cy3-dCTP, respectively, by random primed labeling (Array CGH Genomic Labeling System; Invitrogen, Carlsbad, CA, USA). Hybridizations were performed in a sealed chamber for $48 \mathrm{~h}$ at $37^{\circ} \mathrm{C}$. Subsequent to hybridization, slides were washed according to the manufacturer's instructions and immediately scanned on a GenePix 4200A two-color fluorescence scanner (Axon Instruments, Union City, CA, USA). The acquired images were analyzed using GenePix Pro 4.1 imaging software (Axon Instruments).

Array CGH data analysis. Breakpoint detection and status assignment of the genomic regions were performed using a Gaussian model-based approach (GLAD). The median of the signal ratio (test signal/reference signal) of each triplicated spot was defined as a gain or a loss when it was $>0.25$ or $<-0.25$, respectively. High-level amplification of clones was defined when their intensity ratios were $>1.0$ in $\log _{2}$ scale, and vice versa for homozygous deletion. The threshold value was determined empirically as a value 3 -fold that of the standard deviations calculated from 30 normal males and females in hybridization experiments. The R 2.2.1 package of the Bioconductor Project (http://www.bioconductor.org) was used for the detection of the frequency of gain or loss and for statistical analysis. The Benjamini-Hochberg false discovery rate was applied for multiple testing corrections for the high number of false-positive calls.

\section{Results}

Genome wide array analysis in early GC cases. In total, 22 tumor samples were assessed by high-resolution array $\mathrm{CGH}$ to investigate DNA copy number alterations and new candidate genes associated with early GCs. The majority of clones were frequently gained $\left(\log _{2}\right.$ ratio $\left.>0.25\right)$ or $\operatorname{lost}\left(\log _{2}\right.$ ratio $\left.<-0.25\right)$, with $97.5 \%$ of the clones being gained or lost in $62.7 \%$ of the cases. As the first step of the analysis, chromosome $8 \mathrm{q}$, the most frequently gained [77.3\% (17/22)] and amplified $\left[\log _{2}\right.$ ratio $\left.>1,18.2 \%(4 / 22)\right]$ region in GC cases, was focused upon. There were high-level amplifications on chromosome 8 at four distinct loci, centered at 106.4, 89.6, 77.6 and 110.5 Mb (containing $M Y C$ ). The most common region with copy number increase in GC cases was more clearly defined and narrowed down to 8q22.1-q24.3, encompassing BAC177_N09 to BAC145_J12. A list of the delineations of the 8q22.1-q24.3 chromosomal region and possible target genes of GC is presented in Table I.

Chromosomal alterations of 8q22.1-q24.3 are the most common genetic changes in early GCs. In the 8q22.1-q24.3 region, two common regions of alterations across the chromosome were further clarified. The first loci spanned 96.3-116.8 kb, and was mapped at the 8q22.1-q23.3 region. Notably, two amplified loci in these regions were identified in $9.1 \%(2 / 22)$ of the cases. One locus at $8 \mathrm{q} 22.3$ contained amplified clones covering a region of $\sim 106.4 \mathrm{~kb}$, and comprised the transcription factor CP2-like 3 (TFCP2L3) gene and the other locus spanning $\sim 89.6 \mathrm{~kb}$ on $8 \mathrm{q} 23.1$, without associated genes. The median span of the copy number amplifications was $16.8 \mathrm{~kb}$ (range, 89.6-106.4 kb), and all amplifications were located between BAC157_F12 and BAC169_B05.

The second loci spanned 119.0-144.7 kb on 8q24.11-q24.3, and this region contained 27 possible target genes according 
to the information archived from human genome databases (http://genome.ucsc.edu/). Significantly, a high frequency of copy number gains $\left(\log _{2}\right.$ ratio $\left.>0.25\right)$ and high-level gains $\left(\log _{2}\right.$ ratio $\left.>0.5\right)$ in the $8 \mathrm{q} 24.11-\mathrm{q} 24.3$ region were detected in $77.3(17 / 22)$ and $36.4 \%(8 / 22)$ of the GC cases, respectively. More specifically, two amplified $\left(\log _{2}\right.$ ratio $\left.>1\right)$ loci in these regions were noted in $9.1 \%$ of the GC cases. One locus at $8 \mathrm{q} 24.21$ comprised the representative oncogenes of myelocytomatosis $(M Y C)$ and plasmacytoma variant translocation 1 ( $P V T 1)(4.5 \%)$. The other locus spanning $\sim 110.5 \mathrm{~kb}$ on $8 \mathrm{q} 24.3$ was found to contain cysteine/histidine rich 1 (CYHR1), kinesin family member C2 (KIFC2), forkhead box H1 (FOXH1), protein phosphatase 1 regulatory subunit 16A (PPP1R16A), glutamic-pyruvate transaminase $(G P T), L O C 113655$ and RecQ protein-like 4 (RECQL4) genes with the highest level of amplification. The median span of the copy number amplifications of the 8q24.11-q24.3 region was $32.9 \mathrm{~kb}$ (range, 77.6-110.5 kb), and all amplifications were located between BAC192_N08 and BAC145_J11. An example of an individual profile at the $8 \mathrm{q} 22.1-\mathrm{q} 24.3$ region in the 22 GC cases is shown in Fig. 1. A representative weighted frequency (\%) diagram with high-level amplifications in the 8q22.1-q24.3 region for all 22 GC cases is displayed in Fig. 2.

\section{Discussion}

In the present study, the most notable finding was the frequent genetic abnormality of chromosome $8 \mathrm{q}$ in early GC cases. The long arm of chromosome 8 has long been suspected to contain critical oncogenes that lead to numerous cancer types, including GC (14-17). Abi-Ayad et al (16) reported that copy number gains of the $8 \mathrm{q}$ arm $(70 \%)$ or the entirety of chromosome 8 were the most frequent imbalance in melanoma. de Krijger et al (17) also reported that chromosome $8 \mathrm{q}$ gains were the most frequent genetic abnormality in pleuropulmonary blastoma. Notably, the two surviving patients in the series exhibited gains of chromosome $8 \mathrm{q}$ material as the only genetic abnormality. Furthermore, Buffart et al (14) documented copy number gains of genes on chromosome $8 \mathrm{q}$ in $9.5-73.0 \%$ of the GC cases, with the highest frequency of gains in MYC (73.0\%). More recently, Cheng et al (2) documented the highest frequency (70\%) of copy number gains at $8 \mathrm{q}$ in GCs by array CGH analysis. By combining the results of the present study with those in other studies, it is indicated that copy number gains on chromosome $8 \mathrm{q}$ occur as an early event in the multistage development of various tumors, including GC, which commences in the mildly abnormal epithelium.

More specifically, the smallest region of overlap, the 8q22.11-q24.3 region, was determined. A high frequency of single copy number gains $\left(\log _{2}\right.$ ratio $\left.>0.25\right)$ and high-level gains $\left(\log _{2}\right.$ ratio $\left.>0.5\right)$ in this region was detected in 77.3 and $36.4 \%$ of the cases, respectively. Notably, two amplified $\left(\log _{2}\right.$ ratio $\left.>1\right)$ loci in these regions were detected in $18.2 \%(4 / 22)$ of the GC cases. One locus on $8 \mathrm{q} 24.3$ was found to contain $C Y H R 1, K I F C 2, F O X H 1, P P P 1 R 16 A, G P T$, LOC113655 and RECQL4 genes, placing the highest level of amplifications in the GC cases. To the best of our knowledge, the involvement of these genes in the pathogenesis of GC has not been described previously; however, genetic mutations of these genes are commonly found in various types of cancers. Katoh et al (15) reported high-level amplification and overexpression of the FOXA1 gene in esophageal and lung cancer. Upregulation of the FOXAl gene in pancreatic cancer and basal cell carcinoma, due to the transcriptional regulation by the Sonic Hedgehog ( $\mathrm{SHH}$ ) pathway, has also been documented. Furthermore, overexpression of the RECQL4 gene with chromosomal aberrations and instability in osteosarcoma has also been reported $(18,19)$. These findings indicate that chromosomal alterations of these developmental genes may contribute in playing a role in the underlying mechanism of cancer development.

The other amplified locus at 8q24.21 comprised the representative oncogene of $M Y C$. Several experimental studies have shown that $M Y C$ amplification or overexpression is observed in early GC patients when tumor invasion is confined to the mucosa or submucosa, regardless of the presence of lymph node metastasis $(2,18-22)$. In the study by Onoda et al (20), $M Y C$ expression was found to be more frequent and stronger in early lesions compared with advanced lesions, and Ishii et al (21) also documented increased MYC expression in early GCs in comparison with decreased $M Y C$ expression in late-stage GCs. Based on these findings and the data from the present study, MYC genetic aberrations may be an early event in the pathogenesis of gastric carcinogenesis, and the detection of $M Y C$ locus amplification could be used as an auxiliary tool in GC diagnosis and as a predictor of GC aggressiveness (22).

In addition, one potential oncogene candidate of $P V T 1$ was identified as a potential target within the 8q24.21 amplicon. PVT1 encodes a non-coding RNA and is a host gene for several miRNAs, namely hsa-miR-1204, 1205, 1206 and 1207 (23). Although involvement of the PVT1 gene in the pathogenesis of GC has not been mentioned thus far, genetic mutations of the PVT1 gene have been consistently reported in multiple types of tumors (23-26). Nagoshi et al (24) indicated that PVT1 rearrangements represent a novel molecular paradigm underlying the pathology of 8q24.21 rearrangement-positive multiple myeloma. PVT1 also showed increased expression in prostate cell lines compared to normal prostate tissue (25). In addition, Meyer et al (23) reported that the risk locus can interact with two downstream genes, $M Y C$ and $P V T 1$, and that $P V T 1$, a novel target gene candidate, regulates the $8 \mathrm{q} 24$ risk region. Additionally, an association between genetic variants within PVT1 and Hodgkin's lymphoma has also been postulated (26). Taken together, the results of the present study and the findings of other studies also present evidence that PVT1 is a new target gene candidate, regulated by the $8 \mathrm{q} 24$ risk region, and that it could be defined as an independent target region for chromosome $8 \mathrm{q}$ amplifications in various tumors, including GCs. Further functional and biological studies are required to validate and evaluate the role of the PVT1 gene as a novel candidate oncogene in GC in larger series and on multiple samples.

In summary, the present study confirms and expands upon previous observations that $8 \mathrm{q}$ genetic mutations accumulate early during the multistage pathogenesis of GC. The confirmation of previously reported $8 \mathrm{q}$ gains and the identification of novel target genes at 8q22.1-q24.3 amplified chromosomal sites should provide important clues with regard to the genetic 


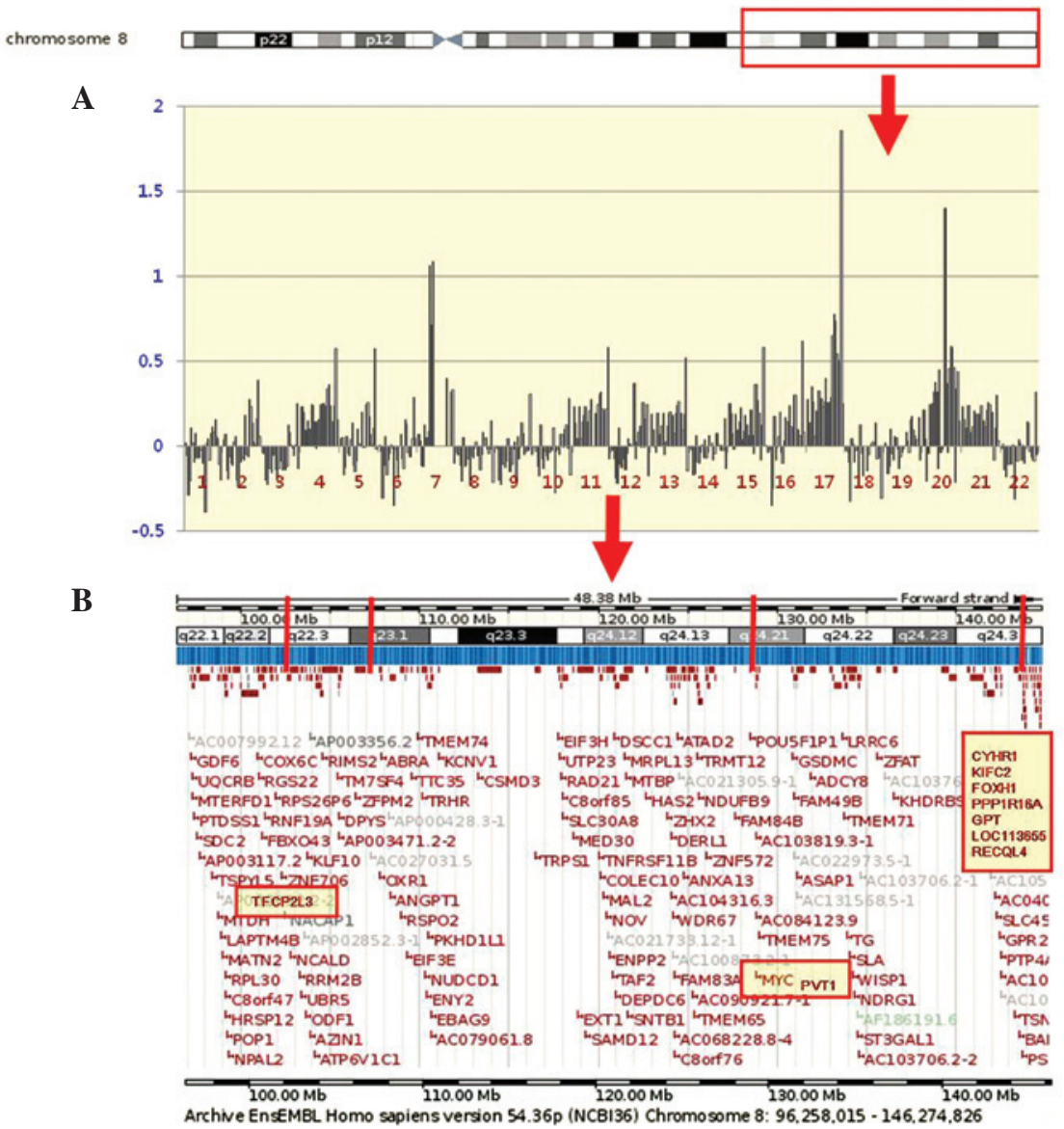

Figure 1. (A) An example of an individual profile at the 8q22.1-q24.3 region in the $22 \mathrm{GC}$ cases. High-level amplifications are clearly seen in cases 7, 17 and 20. Cytobands in the ideogram are shown in the upper image. (B) The schematic presentation of cytogenetic bands, and a map position from the UCSC genome browser is shown below the plot. The candidate target genes (TFCP2L3, MYC, PVT1, CYHR1, KIFC2, FOXH1, PPP1R16A, GPT, LOC113655 and RECQL4) at the 8q22.1-q24.3 region are shaded in yellow. GC, gastric carcinoma; UCSC, University of California Santa Cruz; TFCP2L3, transcription factor CP2-like 3; $M Y C$, myelocytomatosis; $P V T 1$, plasmacytoma variant translocation 1; CYHR1, cysteine/histidine rich 1; KIFC2, kinesin family member C2; FOXH1, forkhead box H1; PPPIR16A, protein phosphatase 1 regulatory subunit 16A; GPT, glutamic-pyruvate transaminase; RECQl4, RecQ protein-like 4.

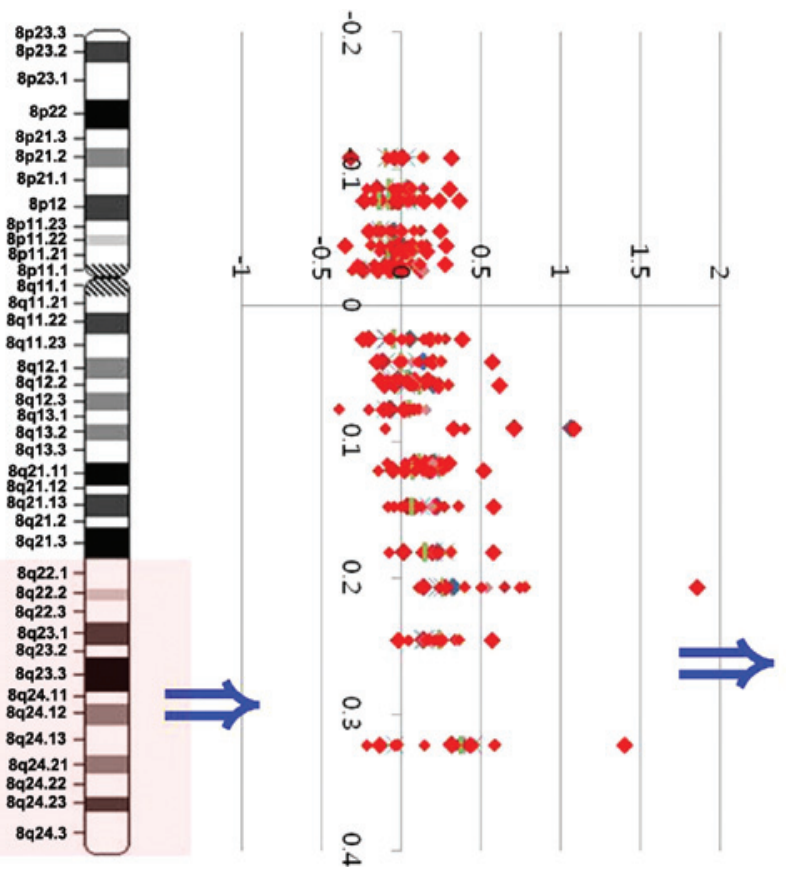

\begin{tabular}{|c|c|c|c|}
\hline & Bac stant. & Bac end & Genecontained In clones \\
\hline 8922.1 & 96258015 & 96396873 & \\
\hline 8922.3 & 102507065 & 102507065 & TFCP2L3 \\
\hline $8 q 23.1$ & 108814200 & 108922667 & MGC35555 \\
\hline $8 q 23.1$ & 107953597 & 108043167 & \\
\hline 8923.3 & 116825035 & 116915090 & \\
\hline $\begin{array}{l}8924.11- \\
8924.12\end{array}$ & 119018437 & 119106743 & EXT1 \\
\hline 8q24.12 & 119283899 & 119352610 & \\
\hline 8q24.12 & 120377587 & 120491239 & NOV, LOC392264 \\
\hline 8924.21 & 128687398 & 128764964 & MYC, PVT1 \\
\hline 8924.21 & 127997591 & 128081281 & Loc401475 \\
\hline 8924.21 & 131222488 & 131303457 & \\
\hline 8924.22 & 134433350 & 134526100 & SIATAA \\
\hline 8924.22 & 134131816 & 134232254 & WISP1, NDRG1 \\
\hline 8924.22 & 133903220 & 133999544 & TG \\
\hline 8924.22 & 136160999 & 136280543 & LOC286094 \\
\hline 8924.22 & 134220116 & 134334477 & NDRG1, LOC392271 \\
\hline $\begin{array}{l}8 \mathrm{qq24.23-}- \\
8 \mathrm{q} 24.3\end{array}$ & 139845147 & 139967047 & COL22AT \\
\hline 8924.3 & 145682925 & 145793387 & $\begin{array}{l}\text { CYHR1, KFFC2, FOXH1, PPP1R16A. } \\
\text { GPT, LOC113655, RECOLA }\end{array}$ \\
\hline 8924.3 & 144691670 & 144777802 & $\begin{array}{l}\text { ZC3HOC3, LOC399693, GSOMDC1, } \\
\text { LOC38964, PP3856, EFFID }\end{array}$ \\
\hline
\end{tabular}

Figure 2. Weighted frequency (\%) diagram of the 8q22.1-q24.3 region in GC cases. In the intensity ratio profiles, the Y-axis represents the map position of the corresponding clone and the intensity ratios are assigned to the X-axis. Cytobands in the ideogram are shown on the left. Genes contained in clones are shown on the right. GC, gastric carcinoma; $M Y C$, myelocytomatosis; $P V T 1$, plasmacytoma variant translocation 1; CYHR1, cysteine/histidine rich 1; KIFC2, kinesin family member C2; FOXH1, forkhead box H1; PPPIR16A, protein phosphatase 1 regulatory subunit 16A; GPT, glutamic-pyruvate transaminase; $R E C Q l 4$, RecQ protein-like 4. 
mechanisms of initiation and progression, and provide new insights into the clinical behavior and management of GC. Additional genome-wide studies with a larger number of patients are warranted to confirm the results of the present study and to improve our understanding of GC.

\section{Acknowledgements}

This study was financially supported by the research fund of Korea Nazarene University in 2013.

\section{References}

1. Nakayama T, Ling ZQ, Mukaisho K, Hattori T and Sugihara H: Lineage analysis of early and advanced tubular adenocarcinomas of the stomach: continuous or discontinuous? BMC Cancer 10: $311,2010$.

2. Cheng L, Wang P, Yang S, et al: Identification of genes with a correlation between copy number and expression in gastric cancer. BMC Med Genomics 5: 14, 2012.

3. Brenner H, Rothenbacher D and Arndt V: Epidemiology of stomach cancer. Methods Mol Biol 472: 467-477, 2009.

4. Gümüs-Akay G, Unal AE, Elhan AH, et al: DNA copy number changes in gastric adenocarcinomas: high resolution-comparative genomic hybridization study in Turkey. Arch Med Res 40: 551-560, 2009.

5. Cheng L, Zhang Q, Yang S, Yang Y, Zhang W, Gao H, Deng X and Zhang Q: A 4-gene panel as a marker at chromosome 8q in Asian gastric cancer patients. Genomics 102: 323-330, 2013.

6. Mihailovici MS, Danciu M, Teleman S, Stanciu C, Stan M, Bălan G and Potoroacă A: Diagnosis of gastric cancer on endobiopsies using the WHO classification. Rev Med Chir Soc Med Nat Iasi 106: 725-729, 2002 (In Romanian).

7. Sun YN and Li Y: Expression of mRNA for membrane-type 1, 2 , and 3 matrix metalloproteinases in human laryngeal cancer. Chin Med Sci J 19: 170-173, 2004.

8. Lowy AM, Clements WM, Bishop J, et al: beta-Catenin/Wnt signaling regulates expression of the membrane type 3 matrix metalloproteinase in gastric cancer. Cancer Res 66: 4734-4741, 2006.

9. Nanjundan M, Nakayama Y, Cheng KW, Lahad J, Liu J, Lu K, Kuo WL, Smith-McCune K, Fishman D, Gray JW and Mills GB Amplification of MDS1/EVI1 and EVI1, located in the 3q26.2 amplicon, is associated with favorable patient prognosis in ovarian cancer. Cancer Res 67: 3074-3084, 2007.

10. Kim KR, Oh SY, Park UC, et al: Gene expression profiling using oligonucleotide microarray in atrophic gastritis and intestinal metaplasia. Korean J Gastroenterol 49: 209-224, 2007 (In Korean).

11. Sanjmyatav J, Steiner T, Wunderlich H, Diegmann J, Gajda M and Junker K: A specific gene expression signature characterizes metastatic potential in clear cell renal cell carcinoma. J Urol 186: 289-294, 2011

12. Hashimoto T, Kusakabe T, Watanabe K, et al: Liver-type fatty acid-binding protein is highly expressed in intestinal metaplasia and in a subset of carcinomas of the stomach without association with the fatty acid synthase status in the carcinoma. Pathobiology 71: $115-122,2004$
13. Hashimoto T, Kusakabe T, Sugino T, et al: Expression of heart-type fatty acid-binding protein in human gastric carcinoma and its association with tumor aggressiveness, metastasis and poor prognosis. Pathobiology 71: 267-273, 2004.

14. Buffart TE, van Grieken NC, Tijssen M, Coffa J, Ylstra B, Grabsch HI, van de Velde CJ, Carvalho B and Meijer GA: High resolution analysis of DNA copy-number aberrations of chromosomes 8, 13, and 20 in gastric cancers. Virchows Arch 455: 213-223, 2009.

15. Katoh M and Katoh M: Human FOX gene family (Review). Int J Oncol 25: 1495-1500, 2004

16. Abi-Ayad N, Couturier J, Devouassoux-Shisheboran M, Grange JD, Kodjikian L and Calender A: Genomic profiling by comparative genomic hybridization: analysis of ten enucleated uveal melanoma cases. J Fr Ophtalmol 34: 17-23, 2011 (In French).

17. de Krijger RR, Claessen SM, van der Ham F, van Unnik AJ, Hulsbergen-van de Kaa CA, van Leuven L, van Noesel M and Speel EJ: Gain of chromosome $8 \mathrm{q}$ is a frequent finding in pleuropulmonary blastoma. Mod Pathol 20: 1191-1199, 2007.

18. Costa Raiol LC, Figueira Silva EC, Mendes da Fonseca D, et al: Interrelationship between MYC gene numerical aberrations and protein expression in individuals from northern Brazil with early gastric adenocarcinoma. Cancer Genet Cytogenet 181: 31-35, 2008.

19. Calcagno DQ, Leal MF, Assumpcao PP, Smith MA and Burbano RR: MYC and gastric adenocarcinoma carcinogenesis. World J Gastroenterol 14: 5962-5968, 2008.

20. Onoda N, Maeda K, Chung YS, Yano Y, Matsui-Yuasa I, Otani S and Sowa M: Overexpression of c-myc messenger RNA in primary and metastatic lesions of carcinoma of the stomach. J Am Coll Surg 182: 55-59, 1996.

21. Ishii H, Gobé G, Kawakubo Y, Sato Y and Ebihara Y: Interrelationship between Epstein-Barr virus infection in gastric carcinomas and the expression of apoptosis-associated proteins. Histopathology 38: 111-119, 2001.

22. Suzuki S, Tenjin T, Watanabe H, Matsushima S, Shibuya T and Tanaka S: Low level c-myc gene amplification in gastric cancer detected by dual color fluorescence in situ hybridization analysis. J Surg Oncol 66: 173-178, 1997.

23. Meyer KB, Maia AT, O'Reilly M, et al: A functional variant at a prostate cancer predisposition locus at $8 \mathrm{q} 24$ is associated with PVT1 expression. PLoS Genet 7: e1002165, 2011.

24. Nagoshi H, Taki T, Hanamura I, et al: Frequent PVT1 rearrangement and novel chimeric genes PVT1-NBEA and PVT1-WWOX occur in multiple myeloma with 8q24 abnormality. Cancer Res 72: 4954-4962, 2012.

25. Jia L, Landan G, Pomerantz M, Jaschek R, Herman P, Reich D, Yan C, Khalid O, Kantoff P, Oh W, Manak JR, Berman BP, Henderson BE, Frenkel B, Haiman CA, Freedman M, Tanay A and Coetzee GA: Functional enhancers at the gene-poor 8q24 cancer-linked locus. PLoS Genet 5: e1000597, 2009.

26. Enciso-Mora V, Broderick P, Ma Y, et al: A genome-wide association study of Hodgkin's lymphoma identifies new susceptibility loci at 2p16.1 (REL), 8q24.21 and 10p14 (GATA3). Nat Genet 42: 1126-1130, 2010 